\title{
Online Tremor Suppression using Electromyography and Low Level Electrical Stimulation
}

\author{
Strahinja Dosen, Member, IEEE, Silvia Muceli, Member, IEEE, Jakob Lund Dideriksen, Member, \\ IEEE, Juan Pablo Romero, Eduardo Rocon, Member, IEEE, Jose Pons, Senior Member, IEEE, Dario \\ Farina*, Senior Member, IEEE
}

\begin{abstract}
Tremor is one of the most prevalent movement disorders. There is a large proportion of patients (around $\mathbf{2 5 \%}$ ) in whom current treatments do not attain a significant tremor reduction. This paper proposes a tremor suppression strategy that detects tremor from the electromyographic signals of the muscles from which tremor originates and counteracts it by delivering electrical stimulation to the antagonist muscles in an out of phase manner. The detection was based on the Iterative Hilbert Transform and stimulation was delivered above the motor threshold (motor stimulation) and below the motor threshold (sensory stimulation). The system was tested on 6 patients with predominant wrist flexion/extension tremor (4 with Parkinson disease and 2 with Essential tremor) and led to an average tremor reduction in the range of $46-81 \%$ and $35-48 \%$ across 5 patients when using the motor and sensory stimulation, respectively. In one patient, the system did not attenuate tremor. These results demonstrate that tremor attenuation might be achieved by delivering electrical stimulation below the motor threshold, preventing muscle fatigue and discomfort for the patients, which sets the basis for the development of an alternative treatment for tremor.
\end{abstract}

Index Terms-tremor suppression, tremor demodulation, neuromodulation, electrical stimulation, afferent stimulation

\section{INTRODUCTION}

$\mathrm{T}$ REMOR is defined as an involuntary, oscillatory movement of a body part. Low-level, so called physiological tremor, is normally present in all persons, but in its pathological form tremor is characterized by high amplitude oscillations at a distinct frequency in the range from 3 to $12 \mathrm{~Hz}$ leading to an impaired motor performance [1]. This

Manuscript received on the $11^{\text {th }}$ of October, 2013. This work has been supported by the Commission of the European Union through the grant ICT2011-287739 (NeuroTREMOR). Asterisk indicates the corresponding author.

Strahinja Dosen, Silvia Muceli, Jakob Lund Dideriksen and Dario Farina are with the Department of Neurorehabilitation Engineering, University Medical Center Göttingen (UMG), Georg-August University, Göttingen, Germany (email: \{strahinja.dosen, silvia.muceli, jakob.dideriksen, dario.farina\}@bccn.uni-goettigen.de).

Eduardo Rocon and Jose Pons are with the Bioengineering Group, Spanish National Research Council, Madrid, Spain (email: \{jose.pons, eduardo.rocon\}@csic.es).

Juan Pablo Romero is with the University Hospital 12 de Octubre, Madrid, Spain (email: juanpa5@hotmail.com). can be a frustrating, embarrassing and debilitating condition; in severe cases, the affected persons are not able to accomplish many of the activities of daily living (e.g., drinking from a glass or bottle). Pathological tremor is considered the most prevalent movement disorder [2].

There are several methods to treat tremor: pharmacotherapy [3], neurosurgery [4], deep brain stimulation [5], mechanical loading and robotics exoskeletons [6]. However, they all suffer from various limitations. Drugs often have side effects [7] and are prone to habituation [8], deep brain stimulators require invasive surgery and increase the risk of brain hemorrhage [9] and/or psychiatric complications [10], while mechanical systems are cumbersome [6] and can interfere with the voluntary movement [11], [12]. Consequently, a universally effective and applicable treatment is still to be found [13], [14].

As tremor is ultimately caused by abnormal muscle activation patterns, Functional Electrical Stimulation (FES) with surface electrodes seems a promising possibility for treatment; it is non-invasive, easily applicable and requires minimal hardware. In FES, the electrodes are placed above the muscles to be activated, and low level electrical current pulses are delivered to the tissue depolarizing the motor nerves and eliciting the muscle contractions. This approach has been already tested in the past, pioneered by Prochazka et al. [15], [16]. They filtered a signal from a displacement sensor to estimate tremor at the wrist and elbow joints and delivered electrical stimulation so that the muscles were activated out of phase with respect to the tremor oscillations. The closed-loop response was tuned so that the system attenuated higher frequencies typical of tremor while minimally influencing lower frequencies characteristic of voluntary movements.

In some recent studies [17], [18], [19], [20] tremor was detected using inertial sensors to measure the kinematics (angle, velocity, and/or acceleration) of the affected joint(s) and extract tremor features (amplitude, frequency). The stimulation was delivered out of phase [17], [18], [19] as in the study of Prochazka et al. [15], [16] or simultaneously to a pair of antagonistic muscles [20] modulating the impedance of the joint to filter out the tremulous oscillations.

Previous studies suggested that surface electromyography signals (EMG) could be used as input for tremor 
detection/prediction and parameter estimation [21], [22], [23], [24]. Therefore, EMG could be used to drive a tremor suppression system as proposed in [19], [25]. This approach has been also tested in simulation studies [26], but the real time implementation and tests in patients are still missing. The only exception is the work of Widjaja et al. [27] who developed an online system for tremor suppression using EMG and accelerometers, which was tested in only one essential tremor (ET) patient.

In all previous studies, electrical stimulation was employed to suppress tremor in a mechanical manner, i.e., by stimulating motor nerves and thereby directly activating muscles, so that the induced muscle forces cancel out the involuntary tremorogenic activation. As the tests in patients demonstrated, this approach is promising but it also has two important drawbacks: 1) prolonged motor stimulation can lead to muscle fatigue [28], and 2) high stimulation intensities necessary to produce muscle contraction can be uncomfortable for the patient. On the other hand, several studies suggested that tremor can be generated, intensified and/or maintained by the pathological activity within the sensory feedback loops. For example, it was demonstrated that tremor in Parkinson patients can be modulated by providing a proprioceptive input induced by passive joint movements [29]. Similarly, electrical stimulation delivered at the wrist joint to median and ulnar nerves modulated the tremor frequency [30]. In a recent simulation study, a neuromusculoskeletal model including several reflex loops (muscle spindles, Golgi tendon organs, Renshaw cells) was used to study the peripheral mechanisms of tremor generation/modulation [31]. It was shown that increasing the gain within the muscle spindle reflex loop can determine the system instability resulting in tremor-like oscillations. The findings of these studies led us to the hypothesis that tremor could be suppressed by stimulating the sensory instead of the motor nerve fibers. In this study, we present a novel system for tremor suppression to test this hypothesis.

The proposed system estimates tremor from the recorded EMG signals online by using an algorithm based on the Iterative Hilbert Transform (IHT) that we have described previously in an offline implementation [24]. Stimulation was delivered over the motor points of the wrist and finger flexors and extensors in an out of phase manner. The system was tested in 6 tremor patients and the results with sensory stimulation were compared with those obtained in the same patients with stimulation above the motor threshold (motor stimulation), considered as the reference standard.

\section{MethodS}

In the sections $\mathrm{A}$ to $\mathrm{C}$, the system components and operation (software and hardware) are presented. Section E contains a detailed description of how the system was applied in the current study (e.g., electrode placement, parameter adjustment, etc.).

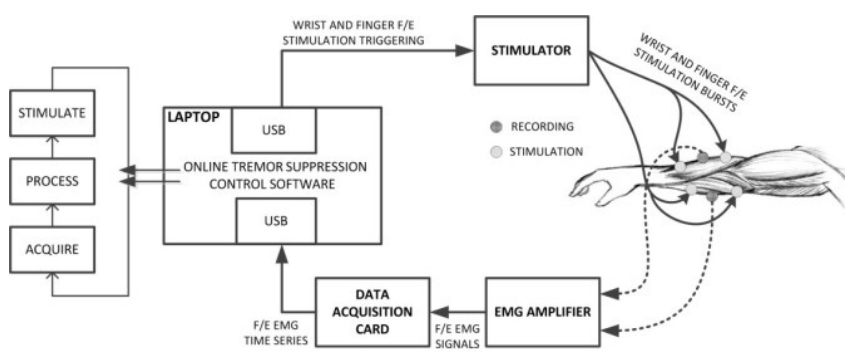

Fig. 1. System components: 1) EMG amplifier, 2) USB data acquisition card, 3) standard laptop with custom-made control software, and 4) programmable stimulator. EMG from the wrist flexors and extensors was recorded, processed to estimate tremorogenic activation, and the stimulation was delivered to the same muscles out of phase with the tremorogenic bursts above and below the motor threshold.

\section{A. System components (hardware)}

The system comprised the following components (Fig. 1): an EMG amplifier (AnEMG12, OTBioelettronica, IT), a stimulator (TremUNA, UNA Systems, SR), a data acquisition card (NI USB 6210, National Instruments, US), and a standard laptop (dual core at $2 \mathrm{GHz}$ ). Two analog bipolar channels and standard $ø 11 \mathrm{~mm} \mathrm{Ag} / \mathrm{AgCl}$ electrodes (Neuroline 720, Ambu, DK) were used to detect EMG from the wrist flexors and extensors. The channels with adjustable gains were equipped with analog band-pass filters with the cut-off frequencies at 10 and $500 \mathrm{~Hz}$. The EMG signals were sampled at $1 \mathrm{kHz}$ by the data acquisition card connected via a USB port to the laptop. The laptop ran custom-made software for tremor suppression, which controlled the acquisition, processed the EMG data and commanded the stimulation unit, as described in "II.B. System operation". The stimulator was battery powered, currentcontrolled and fully programmable; it was connected to the laptop via USB, through which it received textual commands allowing the online control of the stimulation timing and intensity. The USB communication components were optically isolated from the rest of the stimulator electronics. Four stimulation channels in monopolar electrode configuration (common anode) were used to activate wrist and finger flexor and extensor muscles. Disposable square $(5 \mathrm{~cm} \times 5 \mathrm{~cm})$ and round $(\varnothing 3.2 \mathrm{~cm})$ electrodes (PALS Platinum, Axelgaard, US) were used for the stimulation of the wrist flexors/extensors and finger flexors/extensors, respectively. The system was designed for flexion/extension tremor suppression as the muscles/nerves responsible for those movements are superficial and thus suitable for surface detection and stimulation.

\section{B. System operation}

The system implemented a sequential strategy, interleaving recording and stimulation periods (Figs. 2 and 3), i.e., the EMG was captured during the recording time window, the data were processed to determine the presence of tremor and the centers of tremorogenic bursts and based on those, the stimulation timing, and the stimulation was delivered during the stimulation time interval. This strategy was adopted in order to avoid the presence of stimulation artifacts in the EMG signals. The durations of the time windows could be adjusted by the experimenter. The aforementioned steps (i.e., 


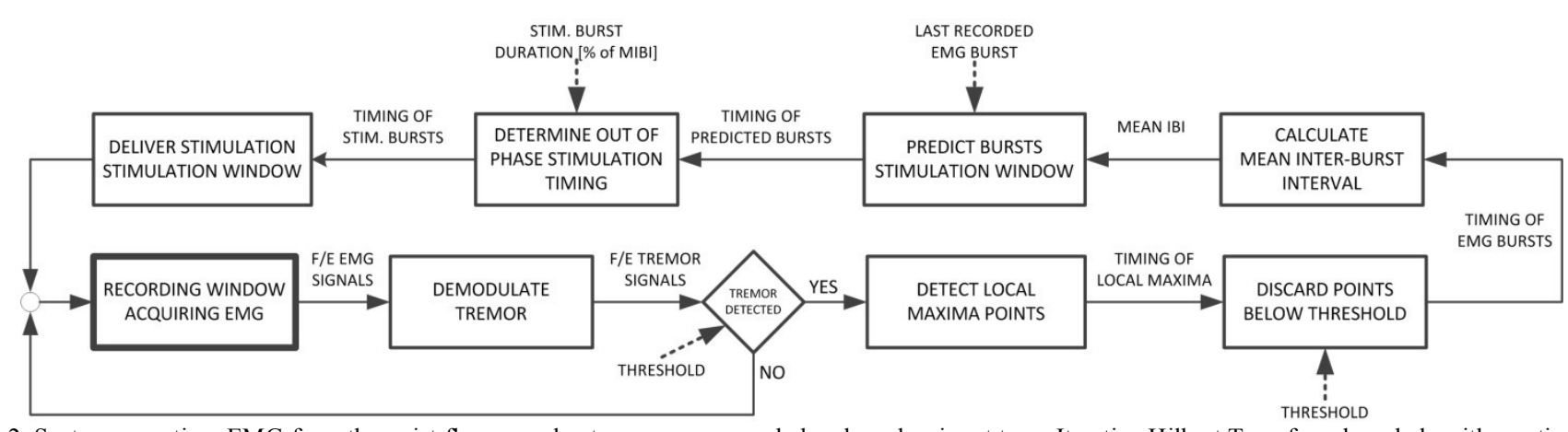

Fig. 2. System operation. EMG from the wrist flexors and extensors was recorded and used as input to an Iterative Hilbert Transform based algorithm estimating the tremorogenic EMG bursts. Based on this, the bursts that were expected to appear within the next stimulation window were predicted and the stimulation was delivered out of phase with the predicted EMG bursts. The rectangles in the figure are the steps of the algorithm, the full arrows represent the data flow, and the dashed arrows are the input parameters. The rectangle with a thick edge denotes the initial step. Notation: MIBI - mean inter-burst interval.
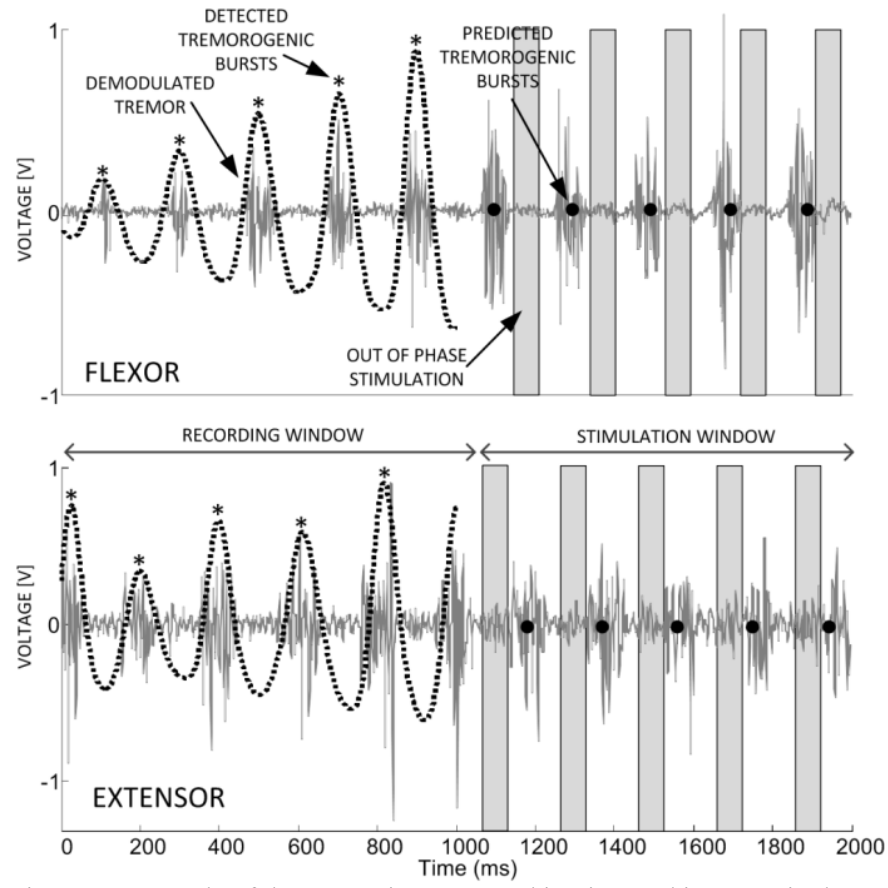

Fig. 3. An example of the processing presented in Fig. 2. This output is shown online to the system operator (see Section II.C). In this particular example, the recording and stimulation windows were 1 second long. The top and bottom panels show the flexor and extensor EMG, respectively, and the dashed line is the estimated tremor demodulated from the EMG. The stars denote the estimated centers of the tremorogenic bursts for the EMG captured during the recording window (tremor signal maxima), and the full black circles are the predicted centers of the tremorogenic bursts within the stimulation window. The grey areas denote the time intervals during which the stimulation bursts are delivered. Note that the occurrences of the predicted bursts match very well those of the actual bursts within the stimulation window, and that the stimulation was delivered out of phase with tremorogenic activation.

recording, processing, and stimulation) were repeated in a loop.

To estimate tremor parameters, we implemented online a recently developed method for tremor demodulation based on the Iterative Hilbert Transform (IHT) [24]. The IHT was applied to the rectified EMG signal, and the first IHT component band-passed between 3 and $12 \mathrm{~Hz}$ was an estimate of the tremorogenic EMG activity (separated from the voluntary component). If the root mean square of the demodulated tremor signal (Fig. 3) was above a user defined threshold (i.e., tremor detection threshold), the tremor was deemed to be present. If the tremor was detected, the centers of tremorogenic EMG bursts were determined by finding the local maxima of the demodulated tremor signal (Fig. 3). Occasionally, there were small oscillations in the tremor signal resulting in local maxima which did not correspond to the tremorogenic EMG bursts. Therefore, in order for a local maximum to qualify as the center of the burst, its value had to be above a user defined threshold (i.e., burst detection threshold). The mean inter-burst interval (MIBI) in the recording window was then calculated, and the subsequent bursts expected to appear within the stimulation window were predicted based on the calculated MIBI and the timing of the last recorded burst. This processing was performed for each muscle group (flexor/extensor) independently. As in the majority of previous tremor suppression studies based on FES [15], [16], [19] the stimulation was delivered out of phase with the estimated tremorogenic activity. The duration of the stimulation bursts was defined manually as a percentage of the MIBI, and the center of the stimulation burst delivered to the flexor (extensor) muscle coincided with the center of the predicted tremorogenic EMG burst of its antagonist, i.e., the extensor (flexor) muscle (out of phase stimulation). Therefore, a single stimulation window for flexor (extensor) muscle included several stimulation bursts, i.e., one stimulation burst centered at each of the predicted tremorogenic extensor (flexor) EMG bursts. The stimulation timing took into account the delays due to signal acquisition and processing. One example of the processing is given in Fig. 3.

\section{Online control software}

The software for online configuration and control of the tremor suppression system was developed in Visual C\# (Visual Studio, Microsoft, US), using Measurement Studio controls (National Instruments, US). The software presented a user friendly interface (Fig. 4) for online system monitoring and setup. The main window comprised a Recording and a Stimulation tab. The Recording tab included the fields for setting up the parameters controlling the EMG acquisition (e.g., sampling rate), tremor demodulation (i.e., thresholds for tremor burst detection) and system operation (e.g., duration of the recording and stimulation/prediction windows, stimulation burst duration in \% of MIBI). The Stimulation tab could be used to select the stimulation channels, adjust the parameters (frequency, pulse width and amplitude) using sliders, and 


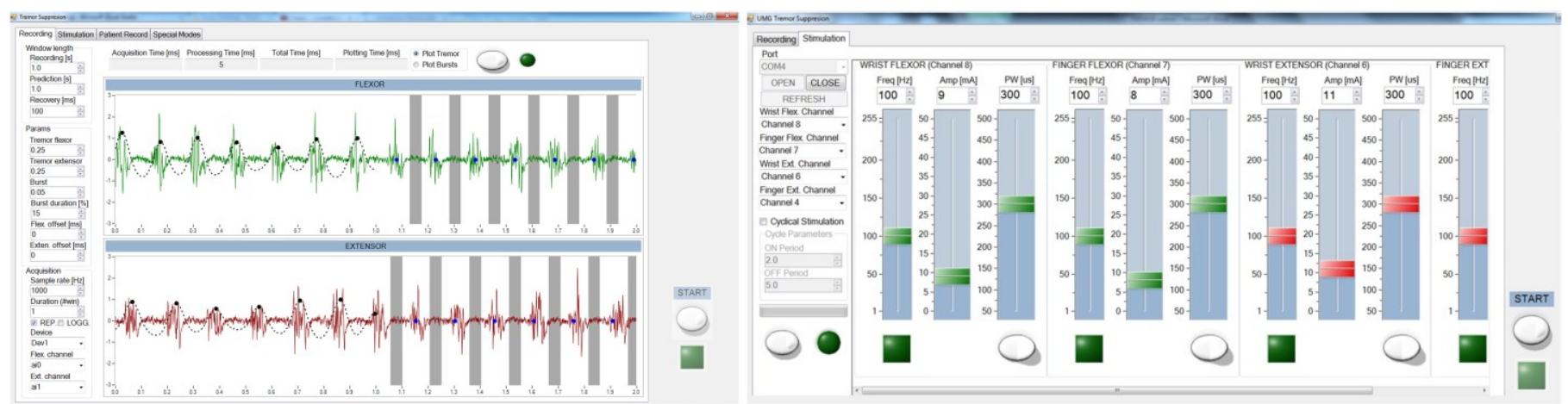

Fig. 4. Software for online tremor suppression: 1) left screen (Recording tab) shows the system online performance (recording, tremor estimation, and stimulation), and 2) right screen (Stimulation tab) displays the controls which regulate stimulation parameters. The graphical interface is user friendly, and allows adjustment of various system and algorithm parameters during the system operation; thereby, the system can be easily customized to each individual subject.

TABLE I

RECRUITED PATIENTS

\begin{tabular}{cccccccc}
\hline \hline Patient ID & Gender & $\begin{array}{c}\text { Age } \\
\text { [years] }\end{array}$ & UPDRSIII & FTMS & Tremor severity & Tremor Frequency [Hz] & Tremor etiology \\
\hline PD1 & Male & 75 & 21 & - & Mild (1) & 3.9 & Parkinson disease \\
PD2 & Male & 58 & 20 & - & Severe (3) & 3.3 & Parkinson disease \\
PD3 & Female & 76 & 23 & - & Severe (3) & 4.2 & Parkinson disease \\
PD4 & Male & 67 & 18 & - & Moderate (2) & 4.2 & Parkinson disease \\
ET1 & Female & 73 & - & 49 & Moderate (2-3) & 4.7 & Essential tremor \\
ET2 & Male & 71 & - & 34 & Moderate (2) & Essential tremor \\
\hline \hline \multicolumn{7}{l}{ Notation: UPDRSII stands for Motor Section III of the Unified Parkinson Disease Rating Scale; FTMS is Fahn-Tolosa-Marin tremor }
\end{tabular}

manually start and stop the stimulation on the specific channel (e.g., for testing the motor points). The system has two operating modes: monitoring and tremor suppression. In the former, the system records the data, displays the processed signals to the experimenter, and depicts the stimulation bursts graphically without delivering the stimulation (Fig. 4, left), i.e., the data for both recording and stimulation windows are shown, where the latter is simulated (no actual stimulation). This mode can be used at the beginning of the session to assess tremor and adjust the system parameters. Once the experimenter is satisfied with the system performance in this mode, the system is switched into the tremor suppression mode. In this mode, the system actually delivers the stimulation to the subject and also reduces the amount of visual output to the experimenter. Specifically, only the EMG acquired during the recording window and the tremor signal demodulated from the EMG are shown, since the EMG recorded during the stimulation window is corrupted by the stimulation artifacts. This output can be used as an indication that the system operates correctly (i.e., periodically acquiring new and valid data).

\section{Subjects}

Six patients were recruited from the Hospital Universitario 12 de Octubre (Madrid, Spain), and clinically examined by the members of their neurological department. Two were diagnosed as having ET and four of them had tremor due to Parkinson Disease (PD). Inclusion criteria included predominant flexion/extension tremor at the wrist. The characteristics of the recruited patients are reported in Table I. The experiments were conducted in accordance with the Declaration of Helsinki and approved by the local ethical committee. All participants signed a written informed consent before the inclusion.

\section{E. Experimental protocol}

The patients were comfortably seated in a chair in front of a desk. In the case of bilateral tremor, the suppression system was applied to the more affected side. The dorsal and volar sides of the forearm were gently cleaned with a wet sponge. The muscle motor points were identified by using a probing electrode (i.e., stimulation electrode with a wet sponge on the top). The electrode was initially placed as recommended in [28] for the stimulation of the wrist and finger flexors and extensors, and then moved around in small steps, until the point resulting with the strongest motor response was identified. Typically, the electrode for wrist extensors was placed close to the later epicondyle of the elbow. For wrist flexors, the electrode was positioned proximal to the elbow crease and somewhat medially. The electrodes for the finger flexors and extensors were located directly distal to those for the wrist muscles. During the probing, the stimulation frequency was set to $100 \mathrm{~Hz}$, the pulse width to $300 \mathrm{us}$, and the current amplitude to a comfortable level producing motor response (flexion/extension of the wrist/fingers). After the electrode location was determined, the self-adhesive stimulation electrodes were placed over the motor points. The neutral electrode $(5 \times 9 \mathrm{~cm})$ was placed at the wrist. Stimulation frequency and current pulse width were set to 100 $\mathrm{Hz}$ and 300 us, and the pulse amplitude was adjusted to obtain a flexion or extension of the joint. As muscle contractions induced by electrical stimulation over the muscle belly are known to be almost exclusively mediated by motor axon recruitment [32], [33] this was referred to as the motor stimulation. To activate selectively the sensory fibers, the current was decreased below the motor threshold (sensory 
stimulation). Starting from the current amplitude determined for motor stimulation, we decreased the amplitude in steps of $1 \mathrm{~mA}$, turned on the stimulation for a few seconds and checked for the signs of motor response. Absence of motor response during the sensory stimulation was verified visually and by palpating muscles and tendons. This was done with one (e.g., wrist flexor) and two channels (e.g., wrist and finger flexor) active, to check for the possible "summation" of the charge in the tissue due to the simultaneous activation of the channels. The electrodes placed over the finger muscles were used only during the sensory stimulation in order to increase the overall recruitment of the sensory fibers, which through the integration at the spinal level might have an effect on the wrist tremor. Motor stimulation was not applied to the finger muscles since the generated forces did not contribute significantly or even had an adverse effect to suppressing tremor at the wrist (pilot tests). Suppressing the finger tremor was outside the scope of the current study.

The skin was prepared for the EMG recordings by gently applying a small amount of abrasive paste. For the wrist extensors, the hand was laid on the table with the palm facing the table surface and the two electrodes were placed next to each other between the stimulation electrodes, approximately halfway along the line connecting the center of the wrist joint and the lateral epicondyle of the elbow. For the wrist flexors, the subject rotated the hand so that the palm was up and the electrodes were placed between the stimulation electrodes, approximately halfway along the line connecting the center of the wrist joint and the medial epicondyle of the elbow. The ground band (circumferential electrode) was secured around the wrist joint, just next to the common anode of the stimulator. The amplifier gain was increased until the tremor EMG bursts became clearly visible. The recording and stimulation windows were set to 1 and $3 \mathrm{~s}$ as in [19], respectively.

Tremor and burst detection thresholds were set initially to $0.25 \mathrm{~V}$ and $0.05 \mathrm{~V}$, which are the values found in pilot tests. The tremor control software was then used in the monitoring mode to assess if the system detected tremor correctly and consistently in a specific patient, and this was checked visually, by comparing the onset of tremor in the patient and the corresponding software output (detected and demodulated tremor). If necessary, the threshold parameters were further adjusted in order to improve the tremor detection. The duration of the stimulation burst was set to $40 \%$ of the MIBI, since this value allowed several pulses to be delivered within a stimulation burst while also assuring that the bursts were well separated. The average tremor frequency in this study was 4 $\mathrm{Hz}$, which means that approximately 10 pulses were delivered per stimulation burst.

To assess tremulous movements, we used an inertial measurement system (XBus kit, XSens, NL) comprising a control box and a set of inertial units (MTx, XSens, NL) measuring the full $3 \mathrm{D}$ orientation of the segments to which they were attached. Two units were strapped to the dorsal sides of the forearm and hand so that the wrist flexion/extension joint angle could be computed. The system performance was assessed by comparing the power of the tremor signals (wrist angle) as measured by the inertial measurement units during the intervals with the system on vs. intervals with the system off (baseline).

Postural tremor is characteristic of ET, and therefore the task for ET patients was to hold the arm outstretched unsupported against gravity [34]. PD patients exhibit tremor mainly at rest, and therefore, these subjects were instructed to position the forearm on a convenient support and relax [34]. In addition, the patients were sometimes engaged in distracting tasks (e.g., speaking, counting backwards, drawing shapes with contralateral hand), since this is known to trigger and/or worsen the tremor.

To prevent fatigue due to keeping the posture and/or stimulation of the muscles at the motor level, the recordings included trials of approximately $2 \mathrm{~min}$ each. Patients were allowed to rest between the trials for a time period under their discretion. The first two trials were used to record the tremor exhibited by the patient while the system was off (baseline tremor, "SYSTEM OFF" condition). Only the portions of the signals with a clearly visible tremor (wrist angle oscillations $>$ $2^{\circ}$ ) were considered. Further trials served to assess the performance of tremor suppression using motor and sensory level stimulation. Within each trial, the inertial measurement system was started first, and once the tremor had developed at approximately the same level as during the baseline recordings, the tremor suppression system was turned on. While the system was active, the electrical stimulation was delivered intermittently, i.e., the recording and stimulation were implemented sequentially (as explained in II.B. "System Operation"). Sensory stimulation was tested first in order to introduce the subjects to the sensation of electrical stimulation gradually, starting with the low intensities. It is known that tremor is very variable phenomenon, and it was sometimes difficult to evoke a consistent tremor in the patients. However, at least five trials of tremor suppression have been recorded for each modality. In total, the measurement session lasted for approximately $2.5 \mathrm{~h}$.

\section{F. Data analysis}

To evaluate the system performance, the segments during which the system was active were extracted from each system test trial and further separated into recording and stimulation intervals. We have compared the following conditions: 1) "SYSTEM ON" vs. "SYSTEM OFF" 2) "SYSTEM ON (STIMULATING)" vs. "SYSTEM OFF", 3) "SYSTEM ON (RECORDING)" vs. "SYSTEM OFF", and 4) "SYSTEM ON (STIMULATING)" vs. "SYSTEM ON (RECORDING)". The goal of the first comparison was to evaluate the amount of tremor suppression while the system was active, sequentially recording and stimulating, with respect to the baseline tremor (system inactive). The second and third comparison assessed the level of tremor suppression separately within the stimulation and recording intervals, respectively. Finally, the two intervals (recording and stimulation) were compared to each other. The construction of the data sets for the analysis is depicted in Fig. 5. We concatenated all the signal segments from one condition, computed the periodogram of the resulting waveform and calculated the tremor power by integrating over the tremor frequencies (between 3 and 12 $\mathrm{Hz}$ ). The average percent suppression was defined as $100 \%$ * 


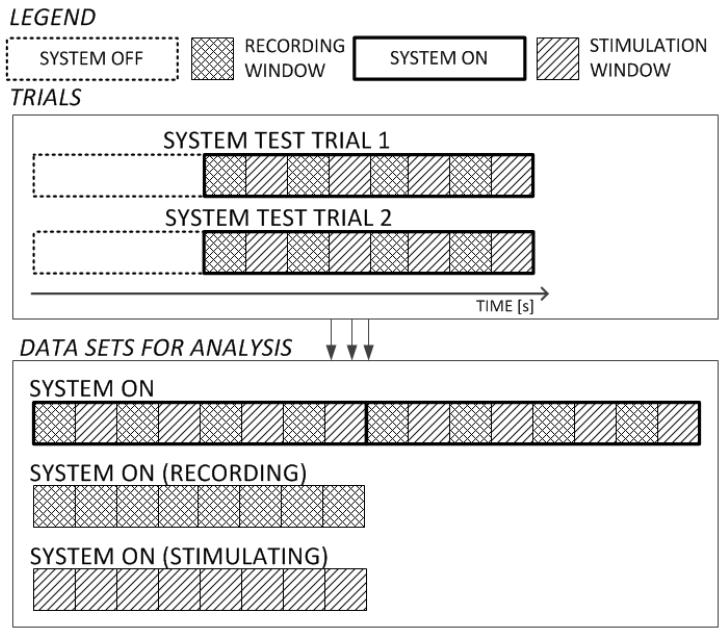

Fig. 5. Organization of the data for the analysis (example for the two system test trials). "SYSTEM ON" data sets were constructed by concatenating all the intervals in which the system was active as extracted from all the system test trials. "SYSTEM ON (RECORDING)" and "SYSTEM ON (STIMULATING)" data sets comprised the data from all the intervals during which the active system was recording and stimulating, respectively.

$(1-\mathrm{PR})$, where the PR (power ratio) is the ratio of the tremor powers in the compared conditions.

One-way repeated measures ANOVA with the stimulation condition as within subjects factor was used to test for the statistically significant difference in the tremor power. In total, there were 7 conditions, i.e., [SENSORY, MOTOR] x [ON, STIMULATION, RECORDING], and SYSTEM OFF. Post hoc analysis (Newman-Keuls test) was applied for the pairwise comparisons. The threshold was adopted at $\mathrm{p}<0.05$.

\section{RESULTS}

\section{A. Representative case}

We first illustrate the performance of the tremor suppression system by presenting detailed results for one patient (ET1). Figure 6 depicts the wrist flexion/extension joint angle recorded by the inertial sensors while the system was active (Fig. 6[a, b]) and while it was off (Fig. 6[c]). In the former case, the system delivered stimulation intermittently, between the recording periods, either below (Fig. 6[a]) or above (Fig. 6[b]) the motor threshold (as described in II.E. "Experimental protocol"). The figure depicts long signals generated by concatenating several trials and provides a global insight into the operation and impact of the tremor suppression system. While the system was off, tremor oscillations were continuous and pronounced, although with a somewhat variable amplitude (peak-to-peak amplitudes between $5^{\circ}$ and $25^{\circ}$ ). Suppression was evident during both motor and sensory stimulation, although the tremor was more attenuated during the motor stimulation.

As it is seen in Fig. 6, the recorded signals (wrist angle) exhibited a characteristic structure during the system operation, i.e., the traces in Fig. 6 are comprised of interleaved segments of higher and lower amplitude wrist oscillations. This reflected a specific pattern of tremor attenuation due to the adopted sequential strategy: tremor was clearly suppressed
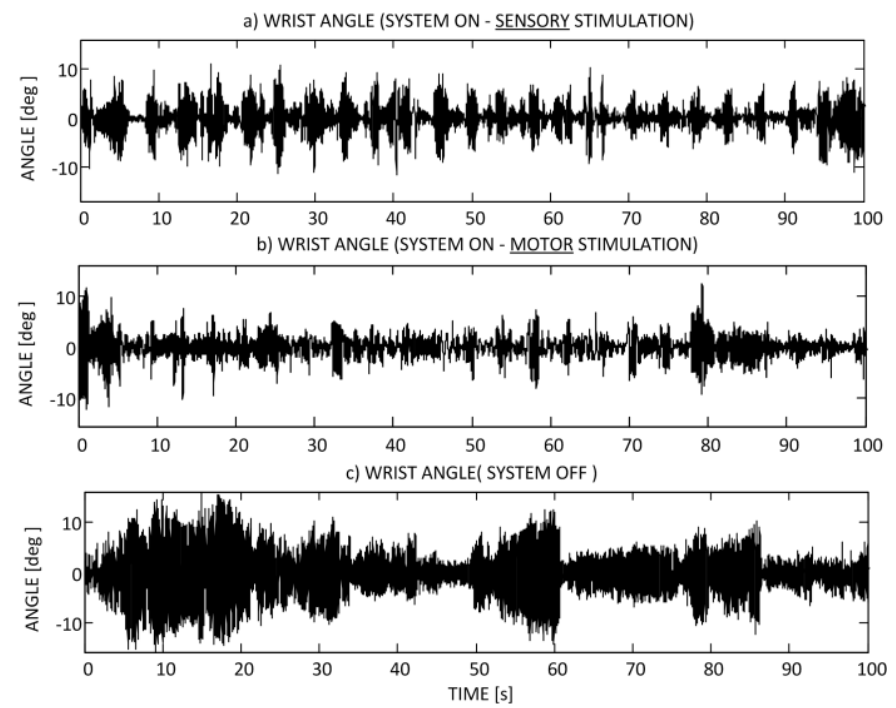

Fig. 6. Wrist flexion/extension joint angle recorded from subject ET1 while the system was active delivering the stimulation below the motor threshold (a), above the motor threshold (b), and while the system was off (c). The signals were created by concatenating data segments from several trials in the aforementioned conditions. Tremor was clearly attenuated while the system was on, with motor stimulation being more effective. Intermittent stimulation produced a burst like structure of the recorded signals in (a) and (b).

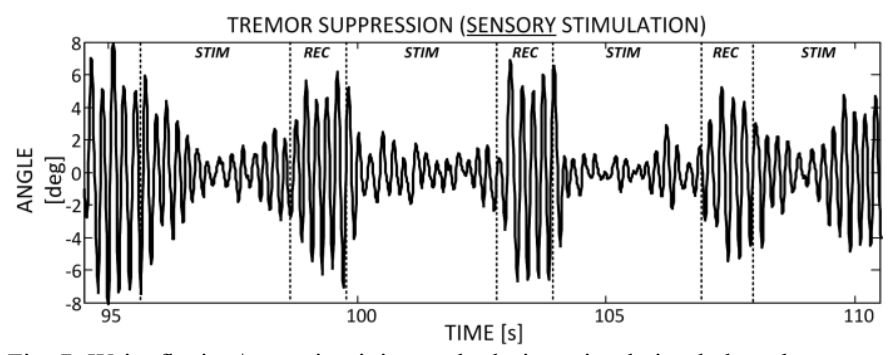

Fig. 7. Wrist flexion/extension joint angle during stimulation below the motor threshold. Stimulation (STIM) and recording (REC) windows are separated by the vertical dashed lines. Note that tremor was attenuated during the stimulation with respect to the recording. The degree and consistency of suppression was however variable.

while the stimulation was being delivered, but then it would recover as the system was recording without stimulating. Figure 7 depicts the wrist flexion/extension joint angle during the recording and stimulation time intervals within a segment of the trial in which the system was active delivering the stimulation below the motor threshold. Tremor was suppressed during the stimulation with respect to recording intervals but the performance was inconsistent. Sometimes tremor was attenuated sharply with the transition between the recording and stimulation, and the attenuation was stable throughout the whole stimulation period (Fig. 7, $2^{\text {nd }}$ and $3^{\text {rd }}$ stimulation interval). Yet, in the very next interval, the amplitude would gradually decrease at the beginning and/or recover to a certain extent towards the end of the stimulation (Fig. $7,1^{\text {st }}$ and $4^{\text {th }}$ stimulation interval).

Frequency analysis (Fig. 8) quantitatively confirmed the qualitative conclusions from Figs. 6 and 7. The drop in the signal power was most pronounced while the system was actually delivering the stimulation (Fig. 8[b, d]). Importantly, although the tremor was re-emerging between the stimulations (i.e., in the recording intervals), it did not recover to the full 

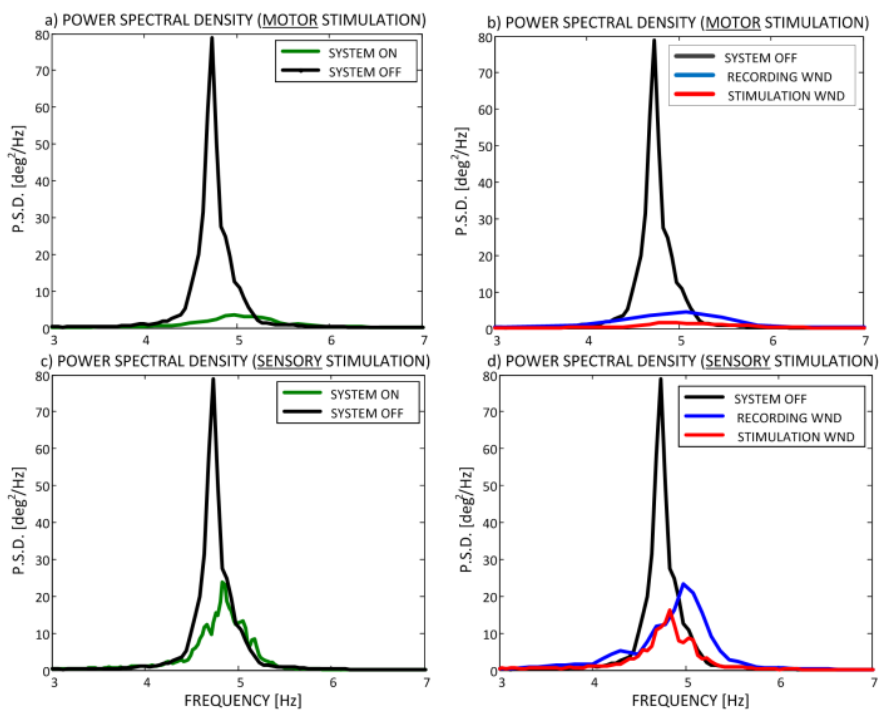

Fig. 8. Frequency analysis of tremor suppression: power spectral density of the wrist angle signals recorded during the delivery of above $([a, b])$ and below ([c, d]) motor threshold stimulation. The plots (b) and (d) compare the tremor power for the signals recorded during the stimulation and recording time intervals separately with respect to the periods when the system was off. The plots (a) and (c) compare the system on (stimulation and recording together) versus system off conditions. There was an overall suppression during both below and above threshold stimulation and during both recording and the actual stimulation delivery.

extent: the average tremor power was lower with respect to the baseline (system off) even when considering only the intervals during which the system was passively recording without stimulating. As a result, there was an overall decrease in the signal power while the system was active, i.e., stimulation and recording taken together vs. system off (Fig. 8[a, c]). Motor stimulation was more successful in suppressing tremor both during stimulation and recording intervals.

\section{B. Overall results}

The levels of tremor suppression for all patients are shown in Table II. We could not suppress tremor in the patient ET2 with either of the two stimulation modalities. This subject was treated as a special case and was therefore excluded from the comparison. While the system was active (stimulation and recording), the average tremor suppression (mean \pm standard deviation) was $60 \pm 14 \%$ and $42 \pm 5 \%$ for above and below motor threshold stimulation, respectively. Both operation modes decreased the power of tremor signal significantly with respect to the baseline $(p<0.01$ for above and $p<0.05$ for below threshold stimulation). Importantly, if only the stimulation intervals were considered, the average suppression for above and below motor threshold stimulation increased to $74 \pm 8 \%$ and $57 \pm 6 \%$, respectively $(\mathrm{p}<0.01$ for above and $\mathrm{p}<$ 0.05 for below threshold stimulation with respect to the baseline). In all the subjects individually and irrespectively of the stimulation modality, tremor power was lower during the stimulation than during the recording, but this was statistically significant overall only for the motor stimulation $(p<0.05)$. If the recording intervals were considered alone, the average results suggested that there was a certain level of suppression with respect to the baseline, but the difference was not statistically significant, likely due to the small number of subjects. For one subject (PD2), when the stimulation was delivered below the motor threshold, the average tremor power during the recording was higher than while the system was off. Finally, although the stimulation above the motor threshold outperformed the below threshold stimulation on average, the statistical tests showed no significant differences between the two. Again, the likely reason for the lack of significance is the small number of subjects. The average stimulation levels were $15 \pm 3 \mathrm{~mA}$ and $18 \pm 5 \mathrm{~mA}$ for the wrist flexor and wrist extensor in the motor stimulation versus $9 \pm 3$ $\mathrm{mA}, 6 \pm 2 \mathrm{~mA}, 10 \pm 3 \mathrm{~mA}$ and $6 \pm 2 \mathrm{~mA}$ for the wrist and finger flexors, and wrist and finger extensors in the sensory stimulation.

\section{Discussion}

We presented a tremor suppression system, which uses a method based on the IHT to estimate tremorogenic EMG activity from the EMG acquired during the recording window, predicts the tremorogenic bursts that will occur within the subsequent stimulation window, and then delivers the stimulation to the antagonistic muscle pair according to this prediction. The control software was developed using a state of the art application development environment (Visual C\#) for good online performance, and also integrates a user friendly graphical interface suitable for the prospective clinical use (see Fig. 4). We tested the system in 6 tremor patients by using two stimulation modalities: above the motor threshold (classic approach) and below the motor threshold (novel approach).

The tests demonstrated that the system was able to substantially attenuate tremor in both stimulation modalities. The suppression while the system was active (recording and stimulation windows) and while the stimulation was being delivered (stimulation windows) was within the values reported previously for motor stimulation $(73 \%$ and $62 \%$ [15], [16], 67\% [19], 52\% [20], and 57\% [27]). The amount of tremor attenuation in the current implementation was limited by the sequential operation of the tremor detection and suppression loops. This strategy was adopted in order to avoid the stimulation artifacts in the EMG. However, with a more sophisticated setup, including an amplifier with a blanking input and a suitable artifact suppression method, the recording, processing and stimulation could possibly be performed concurrently. This would allow the system to reach its full potential and achieve a higher level of tremor suppression consistently and continuously throughout its operation. This would be the level that was achieved in the current tests only intermittently during the actual stimulation time intervals (see Table II). Furthermore, it is possible that the suppression performance may be further enhanced by optimization of the stimulation parameters. To limit the duration of the experimental session for the elderly patients, pulse width and stimulation frequency, known to have an effect on afferent stimulation efficiency [35], [36] were not systematically 
TABLE II

PERCENT TREMOR SUPPRESSION

\begin{tabular}{|c|c|c|c|c|c|}
\hline Patient ID & Stimulation modality & $\begin{array}{l}\text { SYSTEM ON vs. } \\
\text { SYSTEM OFF [\%] }\end{array}$ & $\begin{array}{c}\text { SYSTEM ON } \\
\text { (STIMULATING) vs. } \\
\text { SYSTEM ON } \\
\text { (RECORDING) [\%] } \\
\end{array}$ & $\begin{array}{c}\text { SYSTEM ON } \\
\text { (RECORDING) vs. } \\
\text { SYSTEM OFF [\%] }\end{array}$ & $\begin{array}{c}\text { SYSTEM ON } \\
\text { (STIMULATING) vs. } \\
\text { SYSTEM OFF [\%] }\end{array}$ \\
\hline \multirow{2}{*}{ PD1 } & Motor & 46 & 64 & 4 & 66 \\
\hline & Sensory & 48 & 37 & 32 & 57 \\
\hline \multirow{2}{*}{ PD2 } & Motor & 52 & 58 & 23 & 67 \\
\hline & Sensory & 35 & 73 & -29 & 65 \\
\hline \multirow{2}{*}{ PD3 } & Motor & 53 & 71 & 10 & 74 \\
\hline & Sensory & 44 & 45 & 20 & 56 \\
\hline \multirow{2}{*}{ PD4 } & Motor & 68 & 60 & 42 & 77 \\
\hline & Sensory & 41 & 35 & 21 & 49 \\
\hline \multirow{2}{*}{ ET1 } & Motor & 81 & 61 & 67 & 87 \\
\hline & Sensory & 43 & 49 & 13 & 56 \\
\hline AVERAGE & Motor & $60 \pm 14(* *)$ & $63 \pm 5(*)$ & $30 \pm 25$ & $74 \pm 8(* *)$ \\
\hline$($ mean \pm std $)$ & Sensory & $42 \pm 5(*)$ & $48 \pm 15$ & $12 \pm 24$ & $57 \pm 6(*)$ \\
\hline
\end{tabular}

varied.

In most of the closed loop systems for counteracting tremor based on electrical stimulation, the stimulation was controlled by means of a signal from inertial units. In our system, tremor detection was based on EMG, according to an algorithm we previously proposed and tested offline [24]. As demonstrated in this study, the algorithm can be used for online detection and demodulation of tremor. To the best of our knowledge, only one closed loop system [27] based on tremor detection from EMG has been proposed previously, and it was experimentally tested in only one ET patient. Our system has been tested in a larger population of patients with tremor of different etiologies. The tests showed that the results are generalizable as far as the muscles responsible for tremor are superficial. An important feature of our system is that it can separate the tremulous from the voluntary component of EMG activation. Therefore, the system stimulates only in the presence of tremor and the stimulation time is minimized, lowering the discomfort for the patient and increasing the device battery lifetime.

The main novelty of this study is that it represents a first demonstration that tremor suppression might be achieved by delivering electrical stimulation below the motor threshold, stimulating thereby mainly the sensory fibers. Using sensory stimulation, the tremor was suppressed and the average attenuation degree was not substantially different than with the classic motor approach. The stimulation frequency was higher (@100 Hz) compared to what is usually applied in FES but it was within the range used in neuromodulation applications targeting the sensory pathways (e.g., pain suppression) [37]. It is known that a prolonged higher level motor stimulation at the same frequency would produce muscle fatigue [28], imposing the necessity of increasing the intensity of current delivered. On the other hand, muscle fatigue is not a constraint for the proposed sensory stimulation due to a lack of motor response. Since tremorogenic EMG bursts can be quite short depending on the tremor frequency, we opted for the high frequency stimulation in order to be able to deliver more pulses within each burst, thereby providing a stronger afferent input. The same frequency was then used for the motor suppression in order to compare the two stimulation approaches in conditions that are as similar as possible.

Although the results indicated a reasonable level of suppression on average, the system performance was characterized by a large variability when the sensory stimulation was used. As described in the results, the success in attenuating tremor varied between the patients, between the trials and even between the subsequent stimulation windows of the same patient (Fig. 7). Tremor is a variable phenomenon in general, and to a certain extent this can be reflected in the system performance, but the observed variability could also be explained by an underlying mechanism related to the specific activation of the sensory pathways. Finally, it should be noted that for one patient (ET2), no tremor suppression was attained.

One important methodological concern is that the complete absence of motor response during the sensory stimulation could not be fully asserted. In fact, the motor threshold was determined while the subjects were resting their arms on the table. However, the forearm contains many small muscles whose relative position may change considerably when changing the arm position [38] or when contracting due to the appearance of tremor. It cannot be fully excluded that the same current intensity evoked a small motor response during the tasks performed by the subjects as the nerve can get closer to the electrodes due to relative shift between muscles and electrodes. Nonetheless, the stimulation current was low and we assume that it was too low to suppress tremor mechanically at the levels registered in this study, even if a small contraction was elicited.

The current experiment represents a very first feasibility study for this new neuromodulatory concept of tremor attenuation. The system configuration and stimulation setup have been developed heuristically. Also, since the goal was to compare the motor and sensory stimulation under the same conditions, we have been constrained to use the same protocol for both. The results reported are very promising, but there are many unknowns. Therefore, at this point, we can only speculate about different mechanisms that could be responsible for the attenuation of tremor with sensory stimulation. The observed effect could be due to a direct 
integration of the artificially produced afferent inflow and descending oscillatory commands at the spinal level or due to the projection of afferent input to the supraspinal levels, affecting the actual tremor generating neural networks. Finally, it may also be speculated that the effect is directly related to reciprocal inhibition due to the out of phase time of stimulation. Future work should address these issues by a systematic investigation of this neuromodulation method, including a comprehensive neuromusculoskeletal modeling [39] with peripheral and spinal networks as well as descending input that can be used to simulate tremor and afferent stimulation paradigms. Finally, the trends indicated in the current study are yet to be confirmed in the larger population of subjects.

The sensory stimulation method, if developed further into a consistent and reliable approach, would have several advantages over previous methods. The fact that the stimulation might be effective even when delivered below the motor threshold avoids fatigue and potential pain due to high stimulation intensities typical of other systems. This is even more important in case of elderly patients, who are the majority in the tremor population. The advantages would be most noticeable when the patient is performing daily living activities as sensory stimulation attenuates tremor without interfering with functional movements, contrary to motor stimulation. The system would be thus more comfortable for the patient and more effective in restoring daily task functionality.

The proposed system requires minimal hardware that is easily scalable. In our experiments, we targeted the wrist joint. However, the system can be applied simultaneously to the two joints (e.g., wrist and elbow), as the stimulator features 8 channels and the EMG amplifier 12 channels. The use of EMG as a control signal to drive tremor suppression potentially holds several advantages compared to inertial sensors. First, it allows tremor detection from the muscles directly responsible for the mechanical oscillation ("cause") rather than from the oscillation signal itself ("effect"). Therefore, the corrective action (stimulation) can be tailored to each muscle/muscle group independently for optimizing the tremor compensation. We have indeed observed that tremor can be produced by only one muscle or that different muscles could be producing tremor depending on the current activity of the subject. In these cases, a selective activation might be of interest and a unique feature of the method could be fully exploited by developing an implantable system that would allow very specific activation of sensory-motor structures. Moreover, sensors for EMG detection are placed more proximally than inertial units and are therefore more discrete and less visible, which could improve the cosmetics of the potential future orthotic system.

In its current implementation, the system is designed for the suppression of flexion/extension tremor. However, tremor may occur at multiple degrees of freedom in a joint. For instance, pronation-supination tremor is a typical feature of PD patients [13], [34]. The muscles responsible for those movements are deep and therefore not accessible with surface electrodes. This problem may be solved using intramuscular electrodes for recording/stimulation.

\section{CONCLUSIONS}

In conclusion, a system for suppression of pathological tremor using electrical stimulation of sensory nerves based on EMG recordings was developed and tested on 6 tremor patients. The level of suppression varied across subjects as well as across trials within each patient, but was on average $42 \%$ (recording and stimulation) and 57\% (stimulation only), indicating the potential of the proposed method for implementation in tremor suppression devices. This is the first proof of concept of a peripheral stimulation method for tremor suppression at low intensity stimulation currents, eliciting mainly sensory fibers.

\section{REFERENCES}

[1] R. J. Elble, "Tremor: clinical features, pathophysiology, and treatment.,"Neurol. Clin., vol. 27, no. 3, pp. 679-95, v-vi, Aug. 2009.

[2] G. K. Wenning, S. Kiechl, K. Seppi, J. Müller, B. Högl, M. Saletu, G. Rungger, A. Gasperi, J. Willeit, and W. Poewe, "Prevalence of movement disorders in men and women aged 50-89 years (Bruneck Study cohort): a population-based study.," Lancet Neurol., vol. 4, no. 12, pp. 815-20, Dec. 2005.

[3] K. E. Lyons and R. Pahwa, "Pharmacotherapy of essential tremor: an overview of existing and upcoming agents.," CNS Drugs, vol. 22, no. 12, pp. 1037-45, Jan. 2008.

[4] D. Kondziolka, J. G. Ong, J. Y. K. Lee, R. Y. Moore, J. C. Flickinger, and L. D. Lunsford, "Gamma Knife thalamotomy for essential tremor.," J. Neurosurg., vol. 108, no. 1, pp. 111-7, Jan. 2008.

[5] S. K. Kalia, T. Sankar, and A. M. Lozano, "Deep brain stimulation for Parkinson's disease and other movement disorders.," Curr. Opin. Neurol., vol. 26, no. 4, pp. 374-80, Aug. 2013.

[6] E. Rocon, J. M. Belda-Lois, A. F. Ruiz, M. Manto, J. C. Moreno, and J. L. Pons, "Design and validation of a rehabilitation robotic exoskeleton for tremor assessment and suppression.," IEEE Trans. Neural Syst. Rehabil. Eng., vol. 15, no. 3, pp. 367-78, Sep. 2007.

[7] J. Jankovic, "Complications and limitations of drug therapy for Parkinson's disease.," Neurology, vol. 55, no. 12 Suppl 6, pp. S2-6, Jan. 2000.

[8] J. Jankovic and L. G. Aguilar, "Current approaches to the treatment of Parkinson's disease.," Neuropsychiatr. Dis. Treat., vol. 4, no. 4, pp. 743-57, Aug. 2008.

[9] F. Vergani, A. Landi, D. Pirillo, R. Cilia, A. Antonini, and E. P. Sganzerla, "Surgical, medical, and hardware adverse events in a series of 141 patients undergoing subthalamic deep brain stimulation for Parkinson disease.," World Neurosurg., vol. 73, no. 4, pp. 338-44, Apr. 2010.

[10] S. D. Piasecki and J. W. Jefferson, "Psychiatric complications of deep brain stimulation for Parkinson's disease.," J. Clin. Psychiatry, vol. 65, no. 6, pp. 845-9, Jun. 2004.

[11] E. Rocon, J. Á. Gallego, J. M. Belda-Lois, J. Benito-León, and J. Luis Pons, "Biomechanical loading as an alternative treatment for tremor: a review of two approaches.," Tremor Other Hyperkinet. Mov. (N. Y)., vol. 2, Jan. 2012.

[12] M. J. Rosen, A. S. Arnold, I. J. Baiges, M. L. Aisen, and S. R. Eglowstein, "Design of a controlled-energy-dissipation orthosis (CEDO) for functional suppression of intention tremors.," J. Rehabil. Res. Dev., vol. 32, no. 1, pp. 1-16, Feb. 1995.[13] M. Manto and G. Grimaldi, Tremor: from pathogenesis to treatment. San Rafael, CA: Morgan \& Claypool, 2008.[14]R. J. O'Connor and M. U. Kini, "Nonpharmacological and non-surgical interventions for tremor: a systematic review.," Parkinsonism Relat. Disord., vol. 17, no. 7, pp. 509-15, Aug. 2011. 
[15] A. Prochazka, J. Elek, and M. Javidan, "Attenuation of pathological tremors by functional electrical stimulation. I: Method," Ann. Biomed. Eng., vol. 20, no. 2, pp. 205-224, 1992.

[16] M. Javidan, J. Elek, and A. Prochazka, "Attenuation of pathological tremors by functional electrical stimulation. II: Clinical evaluation," Ann. Biomed. Eng., vol. 20, no. 2, pp. 225-236, 1992.

[17] J. Á. Gallego, J. Ibanez, J. L. Dideriksen, J. I. Serrano, M. D. del Castillo, D. Farina, and E. Rocon, "A Multimodal Human-Robot Interface to Drive a Neuroprosthesis for Tremor Management," IEEE Trans. Syst. Man, Cybern. Part C (Applications Rev., vol. 42, no. 6, pp. 1159-1168, Nov. 2012.

[18] L. Z. Popović, T. B. Sekara, and M. B. Popović, "Adaptive band-pass filter (ABPF) for tremor extraction from inertial sensor data," Comput. Methods Programs Biomed., vol. 99, no. 3, pp. 298-305, Sep. 2010.

[19] L. Popović Maneski, N. Jorgovanović, V. Ilić, S. Došen, T. Keller, M. B. Popović, and D. B. Popović, "Electrical stimulation for the suppression of pathological tremor.," Med. Biol. Eng. Comput., vol. 49, no. 10 , pp. $1187-93$, Oct. 2011.

[20] J. Á. Gallego, E. Rocon, J. M. Belda-Lois, and J. L. Pons, "A neuroprosthesis for tremor management through the control of muscle co-contraction.," J. Neuroeng. Rehabil., vol. 10, p. 36, Jan. 2013.

[21] I. Basu, D. Graupe, D. Tuninetti, P. Shukla, K. V Slavin, L. V. Metman, and D. M. Corcos, "Pathological tremor prediction using surface electromyogram and acceleration: potential use in 'ON-OFF' demand driven deep brain stimulator design.," J. Neural Eng., vol. 10, no. 3, p. 036019, Jun. 2013.

[22] F. Widjaja, C. Y. Shee, W. L. Au, P. Poignet, and W. T. Ang, "An Extended Kalman filtering of accelerometer and surface electromyography data for attenuation of pathological tremor," in 2008 2nd IEEE RAS \& EMBS International Conference on Biomedical Robotics and Biomechatronics, 2008, pp. 193-198.

[23] F. Widjaja, C. Y. Shee, P. Poignet, and W. T. Ang, "Filtering of intended motion for real-time tremor compensation in human upper limb using surface electromyography.," Conf. Proc. IEEE Eng. Med. Biol. Soc., vol. 2009, pp. 2996-9, Jan. 2009.

[24] J. L. Dideriksen, F. Gianfelici, L. Z. P. Maneski, and D. Farina, "EMGBased Characterization of Pathological Tremor Using the Iterated Hilbert Transform," IEEE Trans. Biomed. Eng., vol. 58, no. 10, pp. 2911-2921, Oct. 2011.

[25] D. Zhang and W. T. Ang, "Reciprocal EMG controlled FES for pathological tremor suppression of forearm.," Conf. Proc. IEEE Eng. Med. Biol. Soc., vol. 2007, pp. 4810-3, Jan. 2007.

[26] D. Zhang, P. Poignet, F. Widjaja, and W. Tech Ang, "Neural oscillator based control for pathological tremor suppression via functional electrical stimulation," Control Eng. Pract., vol. 19, no. 1, pp. 74-88, Jan. 2011.

[27] F. Widjaja, C. Y. Shee, W. T. Ang, W. L. Au, and P. Poignet, "Sensing of pathological tremor using surface electromyography and accelerometer for real time attenuation," J. Mech. Med. Biol., vol. 11, no. 05 , pp. 1347-1371, Dec. 2011.

[28] P. L. L. B. (Author), et al PT, Neuro Muscular Electrical Stimulation: A Practical Guide (4th Edition) [STUDENT EDITION], 4th ed. Los Amigos Research Institute, Inc., 2000.

[29] P. M. Rack and H. F. Ross, "The role of reflexes in the resting tremor of Parkinson's disease," Brain A J. Neurol., vol. 109 ( Pt 1, pp. 115-141, Feb. 1986.

[30] J. Spiegel, G. Fuss, C. Krick, K. Schimrigk, and U. Dillmann, "Influence of proprioceptive input on parkinsonian tremor," J. Clin. Neurophysiol. Off. Publ. Am. Electroencephalogr. Soc., vol. 19, no. 1, pp. 84-89, Jan. 2002.

[31] D. Zhang, P. Poignet, A. P. L. Bo, and W. T. Ang, "Exploring peripheral mechanism of tremor on neuromusculoskeletal model: a general simulation study," IEEE Trans. Biomed. Eng., vol. 56, no. 10, pp. 2359-2369, Oct. 2009.

[32] A. J. Bergquist, M. J. Wiest, and D. F. Collins, "Motor unit recruitment when neuromuscular electrical stimulation is applied over a nerve trunk compared with a muscle belly: quadriceps femoris.," J. Appl. Physiol., vol. 113, no. 1, pp. 78-89, Jul. 2012.

[33] A. J. Bergquist, J. M. Clair, and D. F. Collins, "Motor unit recruitment when neuromuscular electrical stimulation is applied over a nerve trunk compared with a muscle belly: triceps surae.," J. Appl. Physiol., vol. 110, no. 3, pp. 627-37, Mar. 2011.
[34] G. Deuschl, P. Bain, and M. Brin, "Consensus statement of the Movement Disorder Society on Tremor. Ad Hoc Scientific Committee.," Mov. Disord., vol. 13 Suppl 3, pp. 2-23, Jan. 1998.

[35] O. Lagerquist and D. F. Collins, "Influence of stimulus pulse width on M-waves, H-reflexes, and torque during tetanic low-intensity neuromuscular stimulation.," Muscle Nerve, vol. 42, no. 6, pp. 886-93, Dec. 2010.

[36] J. M. Clair, J. M. Anderson-Reid, C. M. Graham, and D. F. Collins, "Postactivation depression and recovery of reflex transmission during repetitive electrical stimulation of the human tibial nerve.," $J$. Neurophysiol., vol. 106, no. 1, pp. 184-92, Jul. 2011.

[37] P. Hansson and A. Ekblom, "Transcutaneous electrical nerve stimulation (TENS) as compared to placebo TENS for the relief of acute oro-facial pain.," Pain, vol. 15, no. 2, pp. 157-65, Feb. 1983.

[38] N. Jiang, S. Muceli, B. Graimann, and D. Farina, "Effect of arm position on the prediction of kinematics from EMG in amputees.," Med. Biol. Eng. Comput., vol. 51, no. 1-2, pp. 143-51, Feb. 2013.

[39] F. D. J. Dideriksen, S. Dosen, J. Romero, J. Benito-Leon, J. L. Pons, "Suppression of pathological tremor using electrical stimulation of afferent pathways," in 6th International IEEE EMBS Conference on Neural Engineering, 2013.

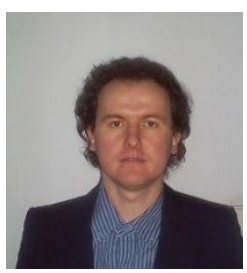

Strahinja Dosen (M'05) received the Diploma of Engineering in electrical engineering and the M.Sc. degree in biomedical engineering in 2000 and 2004, respectively, from the Faculty of Technical Sciences, University of Novi Sad, Serbia, and the Ph.D. degree in biomedical engineering from Aalborg University, Aalborg, Denmark, in 2008. Currently, Mr. Dosen is a Research Scientist at the Department of Neurorehabilitation Engineering, University Medical Center Göttingen (UMG), Göttingen, Germany. His main research interest is the closed loop control of movements and assistive systems. He is a member of the IEEE Engineering in Medicine and Biology Society and the IEEE Computer Society.

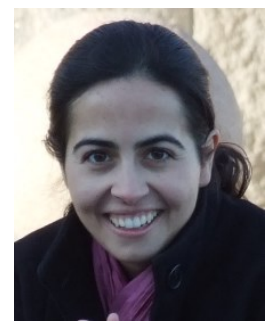

Silvia Muceli (S'09-M'13) received the M.Sc. degrees (cum laude) in Electronics Engineering from the University of Cagliari, Cagliari, Italy, in 2007, and the Ph.D. degree at The International Doctoral School in Biomedical Science and Engineering, Center for Sensory-Motor Interaction (SMI), Aalborg University, Aalborg, Denmark, in 2013. Since 2011, she is working as a researcher at the Department of Neurorehabilitation Engineering, University Medical Center Göttingen, Georg-August University, Germany, within the Bernstein Center for Computational Neuroscience $(\mathrm{BCCN})$. Her main research interests concern surface and intramuscular electromyography, signal processing of biomedical signals and advanced prosthetic control.

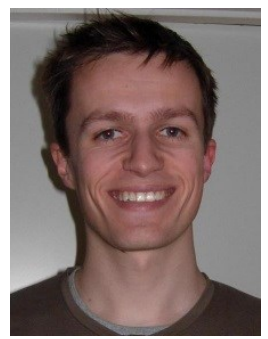

Jakob Lund Dideriksen received the M.Sc. degree in Biomedical Engineering from Aalborg University, Denmark, in 2009 and the Ph.D. degree from The International Doctoral School in Biomedical Science and Engineering, Center for Sensory-Motor Interaction (SMI), Aalborg University, Aalborg, Denmark, in 2012.

Since 2012 he has been working as a researcher at the Department of Neurorehabilitation Engineering, University Medical Center Göttingen, Georg-August University, Germany, within the Bernstein Center for Computational Neuroscience $(\mathrm{BCCN})$. His research interests evolve around the interplay between the central nervous system and the periphery in the control of movements, and how this information can be applied for developing rehabilitation technologies for people with neural or physiological impairments. 


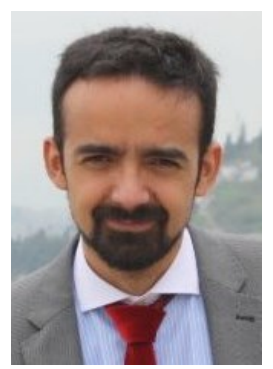

Juan Pablo Romero received the M.D. degree from Central University, Ecuador in 2003 and the M.Sc. degree in Biochemistry and Biotechnology by the Francisco de Vitoria University, Madrid in 2006. He specialized as a Neurologist at the Hospital Universitario 12 de Octubre in 2011 and in 2014 received his $\mathrm{PhD}$ degree in Neuroscience from the Complutense University in Madrid.

He has been an Associate Professor since 2011 of human anatomy at Pharmacy School and Neuro anathomy and Neurology at the Medicine School at the Francisco de Vitoria University, where he has been also the teaching delegate from the Biomedical Research foundation since 2013. He currently works as a Movement Disorders Specialist at Hospital 12 de Octubre in Madrid. His research interest fields are the neurodegenerative diseases, neurophysiology of movement disorders and biotechnological applications for their treatment.

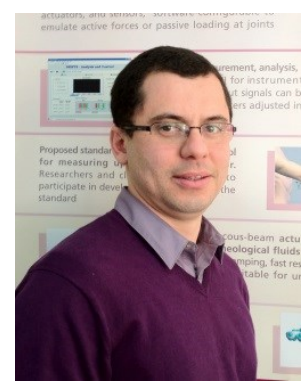

Eduardo Rocon de Lima was born in Vitoria, Brazil (1979). He graduated in Electrical Engineering at Universidade Federal do Espiríto Santo (UFES) in 2001. From 1999 through 2000 he worked as a research associate at Laboratório de Automação Inteligente and successfully held a $\mathrm{CNPq}$ scholarship at UFES. Subsequently he moved to Spain to pursue a Ph.D. degree in Industrial Engineering at Universidad Politécnica de Madrid with Prof. A. Barrientos and Prof. J.L. Pons. His Ph.D. thesis (2006), for which he was awarded the Georges Giralt PhD Award (2008), focused on the development of a rehabilitation robotic exoskeleton that provides a means of testing and validating non grounded control strategies for robotic exoskeletons for active upper limb tremor suppression. Dr. Rocon continued his work in tremor suppression and the application of neuroprosthetics and neurorobotics in rehabilitation on a post-doctoral contract from 2006 to 2009. In 2009, Dr. Rocon was awarded with a Ramón y Cajal contract to continue developing his activities (the most competitive and prestigious postdoc contract in Spain). At the age of 30, Dr. Rocon got a tenured researcher position (2010-present) at the Bioengineering Group at CSIC. His career has recently been awarded the prestigious Juan Lopez de Peñalver Award of the Spanish Royal Academy of Engineering. Dr. Rocon's multidisciplinary work has contributed to different aspects of robotics, neuroscience and medicine. Dr. Rocon research activities have generated more than 40 publications in indexed journals, 1 book, 9 book chapters, more than 100 articles in international conferences, 5 articles in journal of scientific diffusion, and 7 patents.

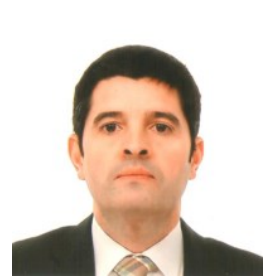

Prof. J.L. Pons has a B.Sc. degree in Mechanical Enginering from (U. Navarra, 1992), a M.Sc. degree in Advanced Control (U. Politécnica de Madrid, 1994) and a Ph.D. in Physics (U. Complutense, 1997). He is with CSIC since 1993 and has actively participated in a number of National, European and International RTD projects in the area of rehabilitation robotics, new actuators and control technologies. In particular, he was scientific coordinator of EU GAIT, EU MANUS, EU ESBiRRo projects, EU TREMOR, EU BETTER and HYPER, all of them focusing on unveiling neuromotors mechanisms in healthy and pathological conditions. He is the editor of Wearable Robotics (John Wiley \& Sons, 2008) a seminal publication in the field on robotics exoskeleton in rehabilitation and assistance. His interests are in the field of rehabilitation robotics, neuroprosthetics and assistive technologies.

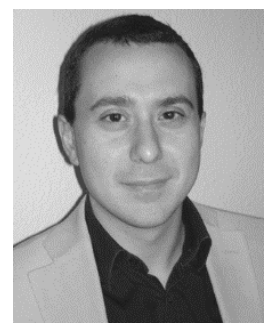

Dario Farina (M'01-SM'09) received the M.Sc. degree in electronics engineering from Politecnico di Torino, Torino, Italy, in 1998, and the Ph.D. degree in automatic control and computer science and in electronics and communications engineering from the Ecole Centrale de Nantes, Nantes, France, and Politecnico di Torino, respectively, in 2002.

During 2002-2004, he was Research Assistant Professor at Politecnico di Torino and in 2004-2008
Associate Professor in Biomedical Engineering at Aalborg University, Aalborg, Denmark. From 2008 to 2010 he was Full Professor in Motor Control and Biomedical Signal Processing and Head of the Research Group on Neural Engineering and Neurophysiology of Movement at Aalborg University. In 2010 he was appointed Full Professor and Founding Chair of the Department of Neurorehabilitation Engineering at the University Medical Center Göttingen, Georg-August University, Germany, within the Bernstein Center for Computational Neuroscience. $\mathrm{He}$ is also the Chair for NeuroInformatics of the Bernstein Focus Neurotechnology Göttingen. His research focuses on biomedical signal processing, modeling, neurorehabilitation technology, and neural control of movement. Within these areas, he has (co)-authored approximately 300 papers in peer-reviewed Journals and over 300 among conference papers/abstracts, book chapters and encyclopedia contributions. Dr. Farina has been the President of the International Society of Electrophysiology and Kinesiology (ISEK) since 2012. He is the recipient of the 2010 IEEE Engineering in Medicine and Biology Society Early Career Achievement Award for his contributions to biomedical signal processing and to electrophysiology and in 2012 he has been elected Fellow of the American Institute for Medical \& Biological Engineering (AIMBE) for his contributions to neurotechnologies. He is an Associate Editor of IEEE Transactions on Biomedical Engineering. 